\title{
Development of guidelines for designing appliances for older persons
}

\author{
Peter G Higgins* and Adam Glasgow \\ Faculty of Engineering and Industrial Sciences, Swinburne University of Technology, PO Box 218, Hawthorn \\ 3122, Australia
}

\begin{abstract}
The ability of older adults to use a domestic appliance depends on their comprehension of the appliance's operation. This may depend on transfer of understanding from similar, more familiar technology. While this may assist their interaction with the new device, it may constrain the discovery of new functionality not represented in the familiar technology, and, thereby, constrain the discovery of advanced functionality. Older adults experience declining cognitive abilities associated with attention, perceptual encoding, and memory (cueing and recall). Designing appliances to extend cognitive abilities provides opportunity to prolong functional independence. Concepts from cognitive psychology, human factors, and gerontology are reviewed to explain age-related behavior towards technology to support innovative product development of technologies for older adults.
\end{abstract}

Keywords: cognitive impairment, domestic appliances, elderly, gerontechnology, human-machine interface, mental model

\section{Introduction}

With age, psychomotor, perceptual, and cognitive abilities decline. Personal independence depends on the ability of seniors to perform instrumental activities of daily living (IADL). Self-reliance depends on their capacity to use domestic appliances such as washing machines, dishwashers, stoves and heaters. Seniors may lack confidence in dealing with new devices: perplexity replacing familiarity. Their inability to adapt leads to increased dependency on family members, friends, and service providers. Where support is scant, seniors may be impelled to move into residential care.

Developing new appliance technologies that compensate for declining abilities may be a means of sustaining self-sufficiency, thereby delaying admittance to residential care. Integrating behavioral science and engineering in a context of product design allows designers to create technologies for supporting and extending declining abilities.

While technology innovation has extended lifespan, there has been little effort in developing technologies that compensate for declining abilities.
Nonetheless, some general guidelines have been developed by Schiebe,r and more recently by Pak and McLaughlin, for designing products for ageing users $[18,25]$.

Disparity between extending lifespan and declining cognitive abilities highlights the need for research into developing technology to sustain independence during cognitive decline [ 32

]. Age-related declines in cognitive abilities are well-documented [25,27]. Older adults experience cognitive decline in attention, perceptual encoding, memory (cueing and recall), and self-efficacy. Cognitive decline can weaken learning and skill acquisition associated with fluid intelligence, spatial ability, perceptual speed, and working memory. There is evidence to show that even healthy older adults exhibit impairment on cognitive tests [1]. Carlson et al. found that executive tests of planning, organization and flexibility to be selectively associated with performance of IADLs [3].

The focus in this paper is the relationship between cognitive impairment and the features of appliance design. Designing appliances to extend cognitive

\footnotetext{
*Corresponding author. E-mail: phiggins@swin.edu.au
} 
abilities provides opportunity to prolong functional independence.

\section{Cognitive capacities related to age}

Age-related deficiencies in cognitive abilities may affect older adults operating semi-familiar or unfamiliar appliances. For example, a person may struggle to use a DVD player in a hotel, when confronted with a remote control that has customary control buttons but an unfamiliar layout. The limited capacity of working memory may constrain the number of perspectives or action sequences that the person may consider [22]. Declines in attention and cognition associated with ageing may restrict capacity. MCI and more severe forms of cognitive impairment (e.g., Alzheimer's disease and Parkinson's disease) may further weaken cognitive abilities, which may vary considerably on a daily basis.

Different cognitive abilities deteriorate at different rates [7]. Inductive reasoning, spatial orientation, verbal memory and perceptual speed, all monotonically decrease to old age from around mid-20s to early 30s [27]. Numeric and verbal abilities both maximize in the mid-40s and then they decrease until the $60 \mathrm{~s}$, at which they plateau.

For appliances used by seniors, designers' attention to the variations in the rates of deterioration in cognitive abilities may be pivotal. The use of multisensory cues in appliances may ameliorate inevitable decline in sensory function. Laurienti et al. found that response times of older listeners provided with redundant visual and auditory cues were equal to those of a young population given only visual cues [12]. They see a practical application of their findings is the use of videophones to aid listener comprehension by affording redundant visual and auditory cues. This stance accords with our conviction to espouse multisensory training strategies to counter age-related functional decline.

\section{Human-appliance interaction}

The creation of new appliance technologies that compensate for declining abilities requires the integration of behavioral science and engineering. Standard texts on human factors engineering, such as Wickens et al. discuss human-machine systems in terms of human-information processing [34].

The cognitive aspects of interaction between a human and an appliance can efficaciously be repre- sented by the model of human information processing in Figure 1. Through their senses, users perceive meaning from the signals from the appliance or the broader environment. The perceptual process depends on sensual input, attention and long-term memory.

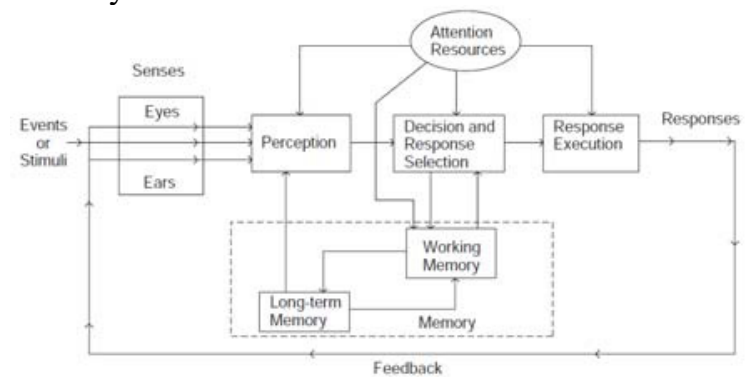

Figure 1. Model of Human Information Processing [31]

\subsection{Senses, perception and attention}

Interaction with appliances is a two-way process in which users send signals to the technology with input devices, commonly called controls, and receive information displayed to them by the technology through their visual, auditory and tactile senses. Information processing is affected by the communication medium: the motor ability of humans in manipulating controls and the performance of visual, auditory and tactile senses in receiving signals from displays. Issues arise regarding the ability of users to signal using the controls (e.g., dexterity in manipulating a control) and in their ability to perceive signals from the technology. Extraction of meaning from the signals received is the process of perception, in which the attribution of meaning derives from experience formed over time. Perception depends on attention and associations drawn from memory. The process of perception concerns bottom-up processing of signals (an ability to resolve the signals relating to factors such as contrast sensitivity and visual acuity) and top-down processing from the users experience, depending on cognitive processes relating to memory and attention.

\subsection{Memory and attention}

Proficiency in using an appliance depends on capacities and capabilities of working and long-term memory and attention resources. The strength of these lessens with age, appreciably because of neurological deterioration.

The working memory of operators affects their aptitude in using functionally complex technological 
devices. The attention-action cycles associated with usage are contingent on users reprocessing information stored in working memory. Response times of users, whether young or old, inflate with increasing task complexity [33]. However, increasing age accentuates this effect, due to both cognitive loading during processing and subsequent temporary storage of information for later processing. When systems are complex, users must recall operations performed, recognize current state of information based on operations already performed, and further apprehend the subsequent operations to achieve their goal. Success and efficiency depends not only on working memory, but also on the ability to inhibit irrelevant stimuli that could influence processing or state recognition. These activities become increasingly difficult as cognitive abilities decline.

As executive control in working memory declines with age, so does functional status measured by IADL [24]. Performance of IADL concerns planning, organization and flexibility, which are functions of executive control. Carlson et al. found that attention is critical for completion of many complex everyday activities [3]. Schieber decomposes attention into four modes [25]. Within these modes, selective and divided attention are of particular interest for the design of appliances to support age-related cognitive impairment.

\subsection{Search and attention}

When using unfamiliar household appliance, users have to find specific controls for particular actions. They must identify these objects from their visual features, formed from primitives, such as shape, color and orientation. Identification depends on their perceptual system extracting the graphical primitives followed by integrating features to create meaningful associations [29]. Basak and Verhaeghen found that the response time for the pre-attentive extraction process does not depend on age [2]. While extraction takes advantage of perceptual automaticity, the integration process relies on selective attention. If target items in visual search have either simple features or features unlike surrounding objects, then searchers can detect the separate features in parallel, without the second serial stage of attention [30]. Older adults can readily identify such targets [20]. For more complex items, focal attention is required for detection of targets-defined by a conjunction of separable properties. The reaction time for this more complex serial processing is proportional to the number of items in the search space and is significantly longer for older than younger adults [20].

\subsection{Inhibiting distractions to attention}

In searching for a specific display element or control, users of an appliance must be able to cope with distractions from task irrelevant information. Inhibitory control over interference from well-practiced extraneous, goal irrelevant, actions decreases with age $[6,35]$. Inhibition depends on the modality of the input and competing limited attentional resources and the processing codes (numeric, textual, spatial). Verhaeghen and Cerella demonstrated that older people find difficulty inhibiting distractions from stimuli that are irrelevant to the prevailing task [33]. If the imposing stimuli concern other tasks that need addressing, they will switch between tasks.

\subsection{Redirecting attention}

Success in using one or more appliances depends on users monitoring performance and actuating controls in response to cues. To respond to a cue, they disengage their attention from the immediate activity and move it to the cue [21]. For example, a grandmother cooking a Christmas dinner for her extended family oversees multiple pots on a stovetop and a roast in the oven. She must respond to cues from sight (bubbling, color), sound and smell, and intervene when necessary (heat adjustment, removal from stove, beginning another subtask, e.g., plating of meal). The complexity involved may overwhelm someone with MCI or more severe impairments.

\section{Appliance use and mental models}

Effective use of an appliance depends upon users understanding the purpose of the device and how it operates. That is, they have some mental representation of both the functions it can perform and the means for accessing this functionality. For users to interact successfully with an appliance, they must recognize that the appliance provides the means for achieving their purpose. Effective operation accords with users forging an appropriate mental model of its operation, in which they can relate the activities they wish to pursue to the functionality of the device.

Ability of older adults to use an appliance depends on their mental model of operation. This may depend on the transfer of understanding from similar, more 
familiar technology. While this may assist their interaction with the new device, it may constrain the discovery of new functionality not represented in the model. Familiar mental models may also interfere with developing appropriate mental models or interaction behavior: for example, a typewriter model may interfere with the formation of a wordprocessing model that includes global find and replace and the application of font and paragraph styles.

When learning to use a new appliance, users may refine their understanding through a trial-and-error exploration of the controls. The discovery process is enhanced if they can relate the product to a familiar mental model of a similar or related product $[5,9]$.

Successful formation and use of mental models depends on persons holding and working with information stored in memory [10]. Once formed in longterm memory, mental models of the functional operation of an appliance may tend to remain static and resistant to alteration until changes to the functionality of the represented system in the real world requires information to be reprocessed in working memory [22]. Clearly, persons with $\mathrm{MCI}$ are at a disadvantage.

Learned behavior from previous technologies may have negative as well as positive consequences. Users may anticipate control structures that create cognitive barriers that restrain seniors exploring new functionality [17]. If users can control basic product functions, they may ignore new functions and features, as their learning requires time and effort. The consequence: an undeveloped mental model of such cognitive tunneling is; the cost: partial use of the product. Moreover, reliance on an inadequate mental model - for example, a model of typewriting used in word processing - may generate a feeling of incapability and a fear of doing something "wrong".

\section{Designing appliances for ageing}

Product designers rarely overtly consider the needs of seniors when designing appliances. Those designers that do so, tend to rely on accessibility guidelines or commonly held presumptions of the needs and limitations of users. To counter unscientific approaches to design, Pak and McLaughlin published a book on designing displays for older adults, with the intention to move designers from compliance with accessibility guidelines to evidence-based research $[18$
]. They focus on the effects of changes in perception, cognition and movement with age.

The creation of domestic appliances that are usable by older persons requires a firm scientific foundation. Factors affecting design listed in the literature for human factors, cognitive psychology and psychophysiology are:

- Decline in visual search and selective attention [20]

- Decline in recognizing targets due to decrease in the ability to extract and integrate features in display objects [25]

- Decline in ability disengage their attention from current activity and redirect it to other display items [21]

- Endurance of lexical and numerical abilities $[7,13]$

Impaired attention affects how many items a person can attend in a display. For example, an older adult seeking the fast-forward button on a DVD player focuses on a suitable target and the number of other items attended in the visual field decreases. This decrease, coupled with an age-related increase in latency of ocular movement affects performance in searching for a specific control in a busy display [26]. To reduce demands on attention, designers should therefore limit the number of perceptual features that users must integrate to discern targets from nontargets [25]. When designing technology for seniors, the lesson for appliance design is to make control and display items clearly different to both one another and the background by lessening shared features. Considering this in the design process may support deeper encoding, thereby making the target selection process more efficient over a shorter period. See Higgins for a discussion on the semantics of visual representations at the interface [8].

Two characteristics of cognitive decline seem paradoxical: difficulty in switching between tasks and easy distraction. To compensate for fixation on the immediate task in situations where users perform multiple tasks contemporaneously (e.g., kitchens), appliances can be designed in a way that accentuates signals from displays based on those cognitive abilities that remain stronger across the lifespan. Different sensory modalities can also be used for competing information streams. In regard to distractions, taskirrelevant information can be removed to minimize clutter from signals that are irrelevant to the current task and likely subsequent tasks [25]. 


\subsubsection{Mental models}

Due to the difficulties older adults experience in attending displays, discerning device features, and processing information in working memory, cognitive workload associated with learning may be excessive. Consequently, the progressive development of well-formed mental models may be hindered. However, if users of the appliance can recognize a familiar functional relationship then their cognitive workload may reduce [28]. That is, by exploiting users' understanding of familiar technologies in appliance design, users may transfer learned patterns of control between old and new technologies [25]. Training can also reduce demands on working memory.

By designing appliances in a way that exploits existing mental models of users, some cognitive load may be shed to externalized prospective memory. In effect, the cognitive system would then combine internalized representations of control and externalized memory aids.

\subsection{Supporting working memory through externalization}

Effectiveness of technology correlates with users accessing appropriate mental models that guide device use. In the case of new devices, successful formation and use of mental models depends on persons holding and working with information in working memory.

Often seniors can cope better if information that they have to remember is externalized. Commonly persons, whether young or old, use external-memory aids for remembering future tasks [14]: For example, jotting notes on a pad or locating objects somewhere that will jog memory. Such practices can counter age-related decline in prospective memory to such an extent that older adults can outperform unaided younger adults $[4,25]$. However, the process of externalizing information requires care. Setting a suitable degree of externalization is difficult, as fixed methods or levels of externalization will not be appropriate for all users. Zhang argues that externalization goes beyond being a mere memory aid: "They are intrinsic components of many cognitive tasks; they guide, constrain, and even determine cognitive behavior" [36]

Designers can provide externalized cues, in the form of Gibsonian affordances, to compensate for cognitive deficiencies by transferring information from memory to the world. Controls or display items on an appliance may provide the means for potential possibilities, but they only become affordances if users realize their potential. A latent predisposition for an applied method of device control (pre-existing mental model) exists when operating a device similar in look or functionality to one a user is familiar. Cognitive workload in using a new product may reduce significantly by exploiting latent predispositions from familiar appliances.

Information that may help older operators use an appliance pertain to the operation's goal, current state, and remaining steps to the goal. Cues for any combination of these may assist older adults control the device. The challenge in designing externalized cues lies in defining the method of signaling and determination of singular or combined forms of cueing that are most effective given the state of cognition of the user.

\subsection{Training}

In discussing cognitive capacities in section 2, we espoused multisensory training strategies to counter age-related functional decline. Displaying control and response stimuli in more than a single sensory mode may help the comprehension of, and reaction to, presented information $[13,19]$.

There are different approaches to learning a new skill. Strategies in developing a skill differ between learning-oriented and performance-oriented persons. Training programs should be directed towards the form of learning orientation. Kozlowski et al contend that the difference is between learning directed towards mastery of the skill itself, versus, activity directed towards demonstrating to others their competency [11]. The former take an active self-motived approach. The latter may avoid situations that challenge their abilities, including training program.

In Georgia, USA, research into the use of Automated Teller Machines by older adults showed that users taught the procedural steps outperformed users undergoing conceptual training. These findings held for both learning and retention over 24 hours. Contrastingly, the type of training experienced by younger users did not affect their performance [16,23]. These studies suggest that instructional design can benefit many older adults in adapting to technologies and new forms of interaction. Moreover, demand on working memory can be significantly reduced through training. Elderly persons may benefit more from training in which they study worked examples instead of conventional means-ends analysis, due to 
task-relevant operations only loading working memory [31].

\section{Adaptive appliance control}

Complex appliances having a high degree of functionality may require users to navigate complex hierarchies of displays using a few controls. Such interaction may be cognitively demanding, as both attention and working memory decline with age. By making everything needed directly visible in the display reduces demands on working memory, as the need to manipulate or transform information in memory is removed $[18,25]$. Such a strategy can be counterproductive, due to increasing demands on visual scanning. Pak and McLaughlin assert that, instead, the challenge is to present information only as needed, thereby minimizing clutter [25]. That is, the display adapts to the direct needs of the user, avoiding the capture of attention by irrelevant stimuli. We contend that such adaptation should also concord with the cognitive abilities of the users. For example, reducing the number of items displayed may reduce interference from distractors, which becomes more challenging as one ages.

Another way that an appliance can be made to adapt concerns a guidance system for the device. Cues that assist the user to recall the interaction process can support older adults in engaging with new appliances that have unfamiliar control structures. By having a different modality for the guidance system to that for controlling the device, the information stream can be segregated from the primary information in the appliance's display. By spreading demands across attentional resources, confusion arising from competing information streams may reduce. The guidance system should adapt to the learning style and knowledge level of the users. User-specific guidance varies with need; lessening or withdrawing as users gain proficiency.

\section{Conclusion}

In developing guidelines for designing appliances for older persons, some general implications can be tentatively drawn from the broad discourse on the relationship between cognitive impairment and the features of appliance design. They are:

- Evoke an appropriate mental model of an appliance's operation by designing the features of graphical objects to reflect, where possible, equivalent objects in similar products

- Make objects in the interface distinctly different from each other and the background

- Concentrate objects that require monitoring within the limited field of view of older persons

- Make the objects in a display immediately accessible and limit information needed for the task at hand

For systems with multiple functions, displays that adapts to the current task the user is performing can remove sources of distraction that may capture attention. Limiting the display to objects for immediate use, maintains the focus of the user on the current task. By adapting human-machine interaction to the cognitive abilities of the users, the degree that the system instructs a user can change with the user's mastery and cognitive capacity. By embodying instructional assistance within appliances, older adults can be guided in using new technologies, with the ultimate purpose of extending their independence at home and maintaining or restoring their self-efficacy.

\section{References}

[1] D.A. Balota, P.O. Dolan and J.M. Duchek, Memory changes in healthy older adults, in: E. Tulving, and F.I.M. Craik, eds., The Oxford handbook of memory, Oxford University Press, Oxford, 2000, pp.395-409.

[2] C. Basak and P. Verhaeghen, Subitizing speed, subitizing range, counting speed, the Stroop effect, and aging: capacity differences and speed equivalence, Psychology and Aging, 18 (2003), 240-249.

[3] M.C. Carlson, L.P. Fried, Q.L. Xue, K. Bandeen-Roche, S.L. Zeger and J. Brandt, Association between executive attention and physical functional performance in community-dwelling older women, Journal of Gerontology: Social Sciences, 54B (1999), S262-S270.

[4] G.O. Einstein, R.E. Smith, M.A. McDaniel and P. Shaw, Aging and prospective memory: the influence of increased task demands at encoding and retrieval. Psychology and Aging, 12 (1997), 479-488.

[5] A. Glasgow and P.G. Higgins, Design of domestic appliances for the cognitively impaired. International Journal of Cognitive Performance Support, (forthcoming).

[6] L. Hasher, R.T. Zacks and C.P. May, Inhibitory control, circadian arousal, and age, in: D. Gopher and A. Koriat, eds., Attention and Performance XVII - Cognitive regulation of performance: Interaction of theory and application, MIT Press Cambridge, MA, 1999, pp.653-675.

[7] C. Hertzog, B.P. Cooper and A.D. Fisk, Aging and individual differences in the development of skilled memory search performance, Psychology and Aging, 11 (1996), 497-520.

[8] P.G. Higgins, Mapping domain semantics to the interface, in: C. Paris, N. Ozkan, S. Howard and S. Lu, eds., Interfacing reality in the new millennium: OzCHI 2000 Conference Proceedings, December 4-8, Sydney, pp.267-274. 
[9] P.G. Higgins and A. Glasgow, Home technology design for the cognitively impaired, in: Proceedings of the 28th Annual European Conference on Cognitive Ergonomics, 25-27 August, Delft, 2010, pp23-26.

[10] P.N. Johnson-Laird, R.M.J. Byrne and W. Schaeken, Propositional reasoning by model, Psychological Review, 99 (1992), 418-439.

[11] S.W.J. Kozlowski, R.J. Toney, M.E. Mullins, D.A. Weissbein, K.G. Brown and B.S. Bell, Developing adaptability: A theory for the design of integrated-embedded training systems, in: E. Salas, ed., Advances in human performance and cognitive engineering research, vol. 1, JAI/Elsevier Science, Amsterdam, 2001, 59-122.

[12] P.J. Laurienti, J.H. Burdette, J.A. Maldjian and M.T. Wallace, Enhanced multisensory integration in older adults. Neurobiology of Aging, 27 (2006), 1155-1163.

[13] R.E. Mayer, Multimedia learning: are we asking the right questions?', Educational Psychologist, 32 (1997), 1-19.

[14] E.A. Maylor, Age and prospective memory', The Quarterly Journal of Experimental Psychology A: Human Experimental Psychology, 42 (1990), 471-493.

[15] S.D., Lima, S. Hale and J. Myerson, How general is general slowing? Evidence from the lexical domain. Psychology and Aging, 6 (1991), 416-425.

[16] S. Mead and A.D. Fisk, Measuring skill acquisition and retention with an ATM simulator: the need for age-specific training. human factors, 40 (1998), 516-524.

[17] N. Moray, Mental models in theory and practice, in: D. Gopher and A. Koriat, eds., Attention and Performance XVII - Cognitive regulation of performance: Interaction of theory and application, MIT Press, Cambridge, MA 1999, pp.223258.

[18] R. Pak and A. McLaughlin, Designing displays for older adults, CRC Press, Florida, 2011.

[19] A. Paivio, Mental representations: a dual coding approach, Oxford University Press, New York, 1986.

[20] D.J. Plude and J.A. Doussard-Roosevelt, Aging, selective attention, and feature integration, Psychology and Aging, 4 (1989), 98-105.

[21] M.I. Posner, A.W. Inhoff, F.J. Friedrich and A. Cohen, Isolating attentional systems: a cognitive-anatomical analysis, Psychobiology, 15 (1997), pp.107-121.

[22] M. Richardson and L. Ball, Internal representations, external representations and ergonomics: towards a theoretical integration, Theoretical Issues in Ergonomics Science, 10 (2009), 335-376.
[23] W.A. Rogers, A.D. Fisk and C. Hertzog, Do abilityperformance relationships differentiate age and practice effects in visual search? Journal of Experimental Psychology Learning Memory and Cognition, 20 (1994), 710-738.

[24] D.R. Royall, R. Palmer, L. Chiodo and M. Polk, Declining executive control in normal aging predicts change in functional status: the freedom house study, Journal of the American Geriatrics Society, 52 (2004), 346-352.

[25] F. Schieber, Human factors and aging: identifying and compensating for age-related deficits in sensory and cognitive function, in: Impact of Technology on Successful Aging, N. Charness and K.W. Schaie, eds., Springer, New York, 2003, pp. $42-84$

[26] C.T. Scialfa, D.W. Kline and B.J. Lyman, Age differences in target identification as a function of retinal location and noise level: Examination of the useful field of view. Psychology and Aging, 2 (1987), 14-19.

[27] K.W. Schaie, The course of adult intellectual development, American Psychologist, 49 (1994), 304-313.

[28] J. Sweller, Cognitive load during problem solving: effects on learning, Cognitive Science, 12 (1988), 257-285.

[29] A.M. Treisman and G. Gelade, A feature-integration theory of attention, Cognitive Psychology, 12 (1980), 97-136.

[30] A. Treisman and J. Souther, Search asymmetry: a diagnostic for preattentive processing of separable features, Journal of Experimental Psychology: General, 114 (1985), 285-310.

[31] P.W.M. Van Gerven, F. Paas, J.J.G. Van Merrienboer and H.G. Schmidt, Cognitive load theory and aging: effects of worked examples on training efficiency, Learning and Instruction, 12 (2002), 87-105.

[32] J.W. Vaupel and A.I. Yashin, Heterogeneity's ruses: Some Surprising effects of selection on population dynamics, The American Statistician, 39, (1985), 176-185.

[33] P. Verhaeghen and J. Cerella, Aging, executive control, and attention: a review of meta-analyses, Neuroscience and $\mathrm{Bi}$ obehavioral Reviews, 26 (2002), 849-857.

[34] C.D. Wickens and C.M. Carswell, Information processing, in: G Salvendy, ed., Handbook of human factors and ergonomics, 3rd edition, 2006, pp.111-149.

[35] N. Wild-Wall, M. Falkenstein and J. Hohnsbein, Flanker interference in young and older participants as reflected in event-related potentials, Brain Research, 1211 (2008), 72-84.

[36] J. Zhang, The nature of external representations in problem solving, Cognitive Science, 21 (1997), 179-217. 